\title{
Understanding the impact of an evidence-based practice curriculum on oral health graduates
}

\author{
G. Wong ${ }^{1}$ M. Print ${ }^{2}$ T. Gerzina ${ }^{1}$
}

\begin{abstract}
Introduction: The explosion of health information accessible to consumers through multimedia has supported the merit and value of evidence-based practice (EBP). Both informed interest from patients and medico-legal considerations of patient care and safety have also contributed to the increased importance of EBP for health practitioners. An EBP curriculum was implemented as part of the Bachelor of Oral Health program at the University of Sydney. The evaluation of student perspectives was previously completed by using questionnaires and focus group interviews. The objectives of this study were to further explore and analyse the potential impact of the EBP curriculum on oral health graduates' clinical practice.

Methods: Semi-structured interviews were conducted to allow for elaboration and clarification to assess oral health graduates' perception of EBP. Graduates $(n=12)$ from both the private and public sectors were invited to participate. Qualitative responses were transcribed and analysed using thematic analysis. Triangulation was used to cross-validate and confirm the findings of previous quantitative data and focus group responses to provide a broader understanding of the subject.

Results: Graduates reported that EBP increased their confidence and empowered them to make clinical decisions. Challenges included limited online resources for literature search, lack of evidence in some aspects of dentistry and difficulty gaining full-text access.

Conclusions: The triangulation approach has enhanced the analysis and interpretation of the impact of the EBP curriculum on oral health graduates' clinical practice. This study has shed light on the possibility of introducing and implementing EBP in other healthcare educational institutions and supports translational science by bridging the gap between research and clinical practice.
\end{abstract}

1 School of Dentistry, Faculty of Medicine and Health, The University of Sydney, Australia

2 School of Education and Social Work, The University of Sydney, Australia

Correspondence

Grace Wong

School of Dentistry

Faculty of Medicine and Health

The University of Sydney

1 Mons Road

Westmead NSW 2145

Australia

Tel: +61288904727

Email: grace.wong@sydney.edu.au 
Keywords: evidence-based practice; triangulation; health education; clinical decision making; qualitative research.

\section{Introduction}

The explosion of health information available to, and accessed by, consumers through multimedia has challenged all health professions regarding the reliability and validity of their service delivery and care of patients. The increasingly "normative" social behaviour of patients to primarily "consult" the internet for health advice strongly supports the importance of an evidence-based approach by health practitioners (Seçkin, Yeatts, Hughes, Hudson, \& Bell, 2016). In applying empirical evidence, health practitioners can informatively and defensibly advise their "informed" patients not only about best practice but also how to discern the validity and quality of multimedia advice (Miles, Loughlin, \& Polychronis, 2007). The value and impact of evidence-based practice (EBP) is demonstrated by its ability to provide justified quality health information to make good health decisions (Gopalakrishnan \& Ganeshkumar, 2013; Schardt, 2011). It appears to be required more urgently now than ever before as a result of enormously increased avenues for "information" and "misinformation". The demand for EBP practice will likely increase in the future.

For any health practitioner, it is challenging to keep up to date with the rapid pace of technological development, new scientific discovery, inventions and innovations that directly impact on current practice. In addition, pressure from professional regulatory bodies and accreditation obligations have also contributed to the increased importance of integrating EBP routinely and regularly in service provision by any health professional in the community. As such, the integration of EBP into clinical practice across all health sectors in the community has become an almost universal expectation (Long, 2003).

Evidence-based medicine (EBM) was originally defined as "the conscientious, explicit, and judicious use of current best evidence in making decisions about the care of the individual patient" (Sackett, Rosenberg, Gray, Haynes, \& Richardson, 1996, para. 3). Vranceanu, Cooper and Ring (2009), however, noted that the initial application of EBM using current evidence alone was inadequate to address patients' problems. Assessment of the patient's circumstances and identification of the best evidence were essential but needed to be considered in conjunction with a patient's characteristics, situation and preferences to finalise a clinical decision (Legare, Ratte, Gravel, \& Graham, 2008; Satterfield et al., 2009). Melnyk et al.'s (2011) definition summed up the concept: "Evidence-based practice is a paradigm and life-long problem-solving approach to clinical decision-making that involves the conscientious use of the best available evidence ... with one's own clinical expertise and patient values and preferences to improve outcomes for individuals, groups, communities, and systems" (p. 575). However, it was evident that the transfer of research findings to clinical practice was, in reality, limited. Effective evidence-based interventions were under-utilised, and there was a delay in translating these interventions to patient care (Glasgow \& Emmons, 2007; Gonzales, Handley, Ackerman, \& O’Sullivan, 2012; Grol, 2001). Despite these limitations, it is important that health professionals possess the skills in appraising and 
apply validly published findings to clinical practice. Researching this problem is the fundamental aim of this study, in which we explore the impact of an EBP curriculum on oral health graduates.

Evidence-based dentistry (EBD) has been included in the University of Sydney Bachelor of Oral Health program curriculum since 2009. Until 2013, the learning content was delivered in Year 2, Semester 1. However, a curriculum review in 2012 determined the delivery of this content was inadequate, as the EBD curriculum only consisted of didactic lectures. It failed to instil the EBP principles and build upon clinical application and, therefore, did not achieve the core objective of EBP, which is translating scientific evidence into clinical practice (Marinho, Richards, \& Niederman, 2001). In 2013-2014, a redesigned EBP curriculum using a multifaceted approach, which was more likely to improve knowledge and skills (Ciarocco, Lewandowski, \& Van Volkom, 2013; Young, Rohwer, Volmink, \& Clarke, 2014), was introduced to the cohort of oral health students during their second year of the undergraduate program. This redesigned curriculum was built upon the existing curriculum by incorporating scenario-based learning (SBL) tutorials to link to clinical practice. It also integrated EBP into student clinical practice, requiring students to demonstrate practice of EBP; incorporated observations of student application of EBP in their interactions with patients; and introduced a progressive assessment strategy to improve student learning through providing formative feedback to students (Saint, Horton, Yool, \& Elliott, 2015). The SBL tutorials actively engaged students in learning EBP application in the classroom (Werb \& Matear, 2004). By integrating EBP in clinical practice, students had the opportunity to step through the process of EBP and apply the best available evidence in practice to their patients (Dawes et al., 2005). Students were assessed on two EBP portfolio cases, receiving constructive feedback for the first case before submitting the second case. The process evaluation was conducted using questionnaires to assess student perspectives at the end of year 2. Focus groups were conducted to further explore students' learning experiences at the end of year 3 .

Research on EBP in Australia is limited. Earlier reporting of this research study, using the quantitative results, found all students $(n=62,2013 \& 2014$ cohorts $)$ agreed that EBP influenced their clinical decisions. There was a significant positive correlation between teaching EBP in clinical practice and students' perceived confidence in the EBP process (G. Wong, Monatzerolghaem, \& Gerzina, 2018). The majority of oral health students agreed EBP should be an integral part of clinical practice. From the focus group responses, the participants appeared to understand the need to look for evidence that helped to confirm and justify clinical decision making. They demonstrated positive attitudes towards EBP and used various words to describe their experience, such as "beneficial", "essential", "motivate" and "keep up to date". Figure 1 summarises the emerging themes from the focus group discussions.

There are various studies integrating EBP into the curriculum in different health professions, including the dental curriculum (Azarpazhooh, Mayhall, \& Leake, 2008; Gerzina, McLean, \& Fairley, 2005; Kersten, Vervoorn, Zijlstra, Blok, \& van Eijden, 2007; Teich, Demko, \& Lang, 2013); however, there is no research to date studying 


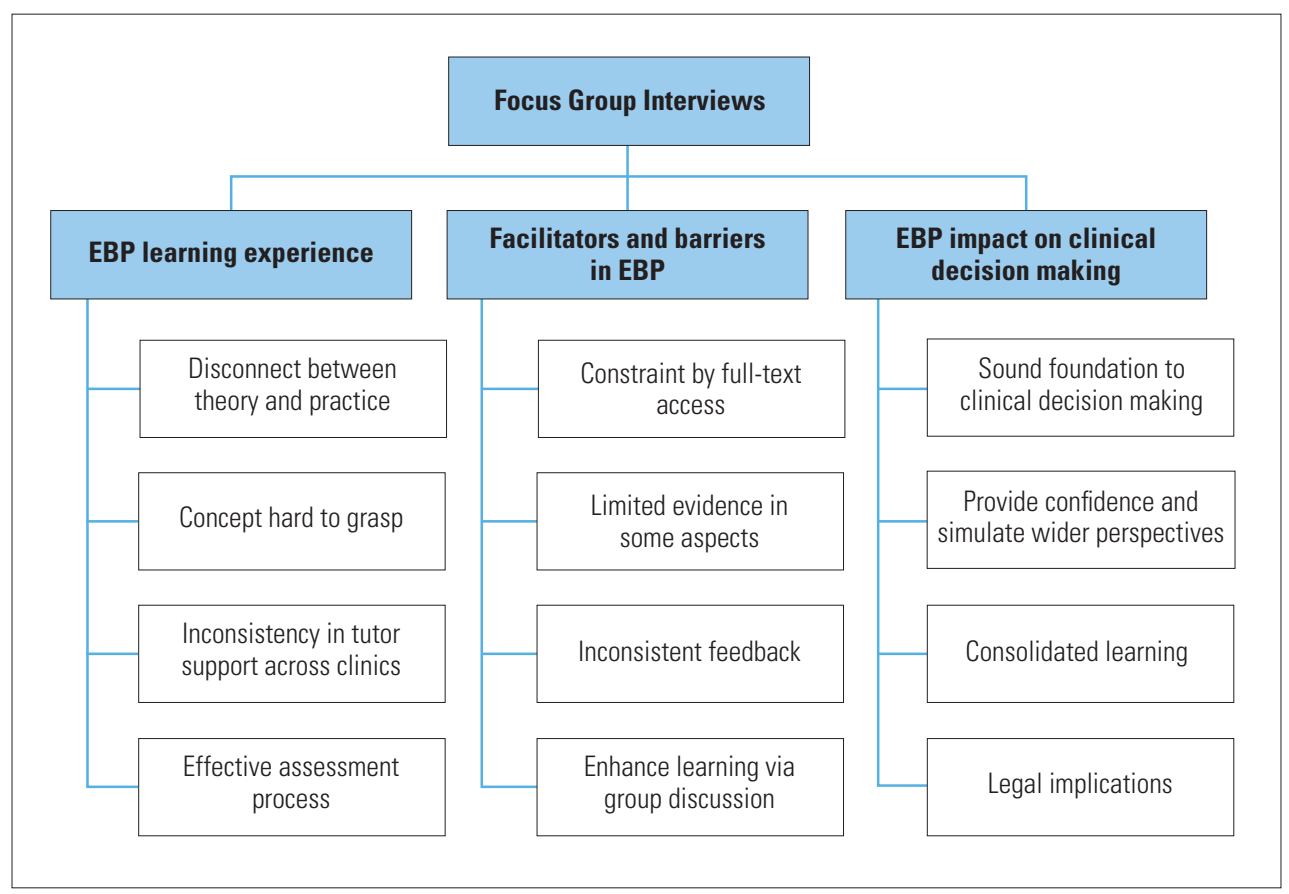

Figure 1. Summary of themes from focus groups.

an EBP curriculum within an undergraduate oral health curriculum and its impact. Elaborating on the aim of this study, our intention is further in-depth exploration of the University of Sydney oral health graduates' perceptions of the impact of EBP curriculum on career practice. This led to multiple research questions, but for this study, the focus was the relationship between EBP and graduates clinical practice. The research questions included:

1. What was the graduates' overall perception of EBP?

2. How effectively was EBP implemented by graduates in their clinical practice?

3. How effectively was EBP supported in graduates' clinical practice?

4. What was the graduates' overall perception of their experience of EBP teaching within the curriculum?

This research project was approved by the University of Sydney Human Research Ethics Committee (2013/1066). Informed consent forms were distributed to participants to indicate their voluntary participation in the project. 


\section{Methods}

\section{Phenomenology and data collection}

Phenomenology as a research design seeks to explain particular phenomena through the study of structures of consciousness as experienced from the first-person point of view. "The central structure of an experience is its intentionality, its being directed toward something, as it is an experience of or about some subject" (Smith, 2013, para. 1). In this case, we are considering the experience of participating in EBP. An explanatory model of a sequential mixed-method design was used. In the earlier phase of the project, quantitative data was collected first, using a questionnaire, and then explored during a second qualitative phase, which further explained the quantitative results (Creswell, 2013; Duffy, 1987; Foss \& Ellefsen, 2002). The questionnaire and focus group data were reported in the introduction section. For this phase of the study, data collection involved individual interviews with oral health graduates. The interview questions delved into the impact of EBP in the oral health profession. Phenomenology was used to encourage participants to conceptualise and describe the phenomenon of their personal experience in EBP (Starks \& Trinidad, 2007). The goal of this research method was to maximise the depth of information collected; therefore, semi-structured one-on-one interviews were conducted with individual participants. The interviews were designed to gather information about graduates' perception of EBP with respect to four main areas, which helped to answer our first research question.

\section{Participant demographics}

Nested sequential data sampling was chosen for this mixed-methods research. The participants in the qualitative component, which included focus groups and interviews, were selected from the participants of the quantitative study (Teddlie \& Tashakkori, 2011). Twelve participants from the graduate cohort were purposefully invited, by email, to participate in this qualitative study. In order to maintain homogeneity of the graduates for the one-on-one interviews, the recruitment was restricted to only graduates who had worked continuously full-time for 12 months within one practice. Those graduates who had less than 12 months working experience were excluded from this study. This was to ensure that the selected participants were especially experienced with the phenomenon of interest, evidence-based practice, and who were able to provide information-rich, in-depth responses (Palinkas et al., 2015). Sixteen potential participants were contacted and 12 fulfilled the inclusion criteria. Selection for the participants was based on their practice location (rural and metropolitan districts, private dental practices and public dental hospitals). Six graduates worked in the private sector in the metropolitan area of Sydney. Five graduates worked in the public sector in rural New South Wales, while only one worked in the metropolitan area. Table 1 shows characteristics of the participants. 
Table 1

Characteristics of Interview Participants

\begin{tabular}{|c|c|c|c|c|}
\hline & Private & Public & Metro & Rural \\
\hline Participant 1 & $\checkmark$ & & 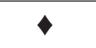 & \\
\hline Participant 2 & $\downarrow$ & & 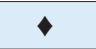 & \\
\hline Participant 3 & & $\downarrow$ & & $\diamond$ \\
\hline Participant 4 & & 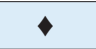 & & 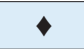 \\
\hline Participant 5 & & $\checkmark$ & & $\checkmark$ \\
\hline Participant 6 & & 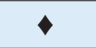 & & $\checkmark$ \\
\hline Participant 7 & & 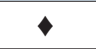 & 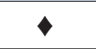 & \\
\hline Participant 8 & 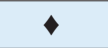 & & 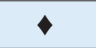 & \\
\hline Participant 9 & & 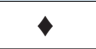 & & 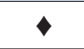 \\
\hline Participant 10 & $\checkmark$ & & $\downarrow$ & \\
\hline Participant 11 & 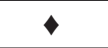 & & 4 & \\
\hline Participant 12 & 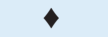 & & $\bullet$ & \\
\hline
\end{tabular}

\section{Analysis of transcripts and assessors}

Twelve broad questions were developed to direct interviews (Figure 2). The questions were open ended in a conversational format. The investigator asked the same questions of all participants, but the order of the questions, the exact wording and the type of follow-up questions varied. One-on-one semi-structured interviews were conducted, which lasted 30-45 minutes. All interviews were digitally recorded to preserve the entire verbal part of the interview for later analysis, and the recordings were transcribed without mention of any of the participants' names. A thematic analysis of the interview transcripts, using coding techniques, was completed by the investigators and independently by assessors invited to the project. The three assessors did not report any conflict of interest in participating in the study and were aware that choosing whether to participate or not had no advantage or disadvantage for them. The assessors were provided with an explanation of the nature of the study, the data structure and the rationale behind the team approach to coding. They then applied an open, interpretative, non-directed process to the transcript. The emerging themes were then compared and verified with the assessors, who selected several quotations that supported their choice of themes to include in the results. For ease of reading, grammatical inconsistencies were corrected, but no substantial changes were made to the assessors' analysis.

\section{Triangulation}

In order to enhance the validity of this research, methodological triangulation using mixed methods was applied to evaluate the strategies and impact of the EBP curriculum on oral health graduates (Farmer, Robinson, Elliott, \& Eyles, 2006). The quantitative results were presented in a previous study (G. Wong et al., 2018), and qualitative results 


\section{Graduate interview questions}

Questions on assessment of evidence-based practice:

1. What is your assessment of evidence-based practice for everyday work since you graduated?

2. How do you feel about the need for integrating evidence-based dentistry into your day-to-day clinical practice?

3. What do you see as the advantages of evidence-based practice?

4. What is your view on the shortcomings of evidence-based practice?

Questions on implementation of evidence-based practice:

5. To what degree has evidence-based practice changed your clinical decisions?

6. How does your evidence-based practice influence your patient's preferences?

7. Give details if you have experienced and problems/issues in implementing evidence-based dentistry in your clinical practice.

Questions on support for evidence-based practice:

8. What level of support do you have from your supervisor/manager for evidence-based practice?

9. Tell me the support you have from your colleagues for evidence-based practice.

10. What level of support do you have at work for using online resources?

Question on evidence-based teaching at $\mathrm{BOH}$ :

11. Did the EBP teaching at $\mathrm{BOH}$ provide you with adequate skills in implementing evidence-based practice? Explain its adequacy or inadequacy.

Question on evidence-based teaching at $\mathrm{BOH}$ :

12. How do you see yourself in the future using evidence-based practice?

Figure 2. Graduate interview questions.

of student focus groups and individual interviews of the graduates would form a more complete picture of the impact of the EBP curriculum.

Data source triangulation can provide better insights and verify findings by collecting data from different groups (Carter, Bryant-Lukosius, DiCenso, Blythe, \& Neville, 2014; Sargeant, 2012). Focus groups were conducted to examine the views of two different groups of students: high school leavers and graduate students (students who had completed another degree before entering the University of Sydney Bachelor of Oral Health program. The graduate students held opinions that were comparatively different from those of the high school leavers. Individual interviews were conducted to 
examine the perceptions of two different groups of graduates in different settings within the oral health profession: graduates working in the private sector and those working in the public sector.

\section{Results}

The investigators met with the assessors to discuss and challenge the assessors' data, with the aim of arriving at common themes, which emerged as conclusions in this consensus discussion. Figure 3 summarises the themes from the graduate interviews.

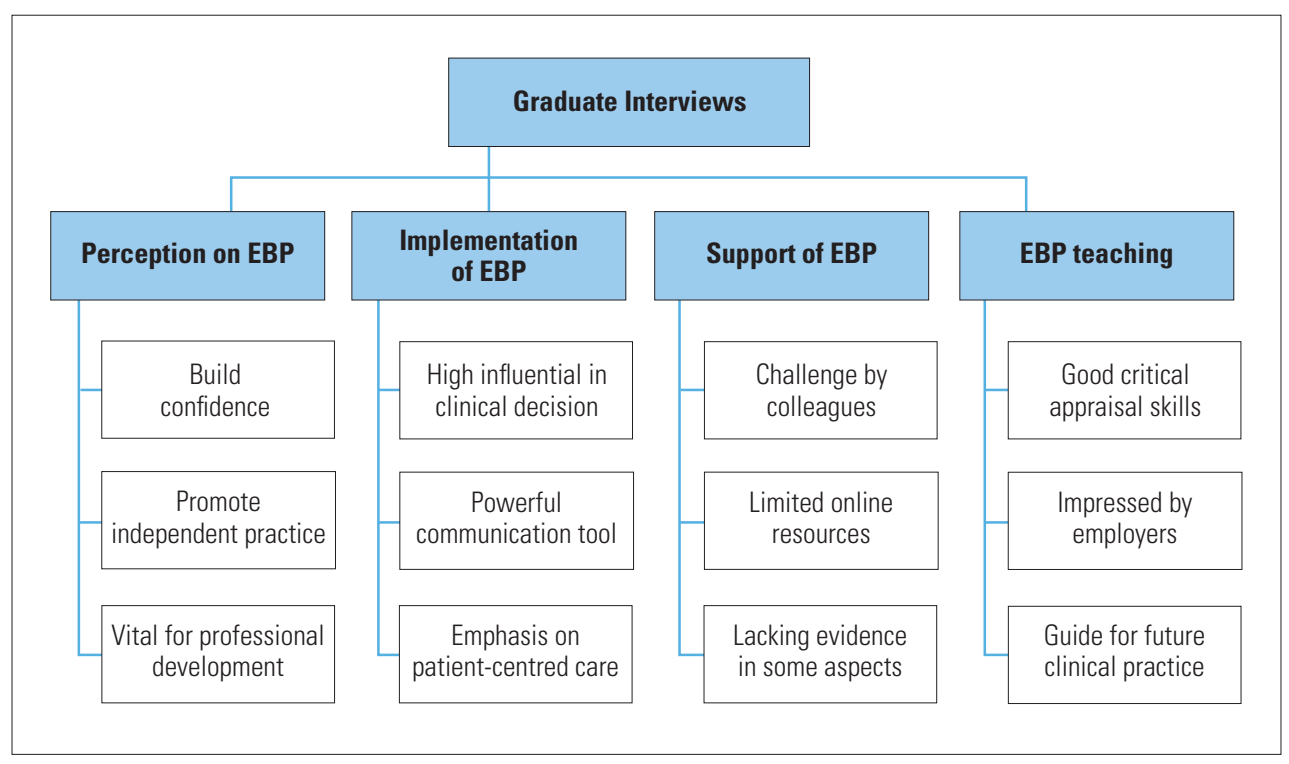

Figure 3. Summary of themes from the graduate interviews.

\section{Graduates' overall perception of EBP}

\section{Building confidence}

Both groups of oral health graduates, whether they worked in the public or the private sector, felt that EBP is vital in building confidence in clinical practice. Illustrative comments included (verbatim):

It certainly had a solid impact in my practice. It stopped me from second-guessing. (Participant 8, private, metro)

At first I feel intimated in front of an experienced clinician, but with EBP, I don't feel pressured to bow down to "old" theories and methods. (Participant 2, private, metro) 


\section{Promote independent practice}

While the oral health graduates work within a structured professional relationship with their employer dentists, they exercise autonomous decision making within their particular areas of competence to provide the best possible care to patients. In the interview, graduates claimed that EBP helped them make independent clinical decisions with their patients.

EBP was vital to my practice as I worked in rural clinics. I didn't have the luxury to ask a dentist all the time. Looking up evidence gave me the confidence to work independently. (Participant 5, public, rural )

As our profession has moved to become a more independent health practice, we are no longer working under the supervision of dentists. Evidence-based practice would certainly be the best support to work independently. (Participant 7, public, metro)

Vital to professional development

All respondents agreed that it was important to keep up to date with the latest evidence on new theories and new products so they could provide the best treatment options for their patients.

[It] makes you a better clinician if you can keep up with EBP, so you are growing in your profession. (Participant 4, public, rural)

EBP plays a critical role in continuing professional development; I would certainly keep up for the sake of best patient care. (Participant 9, public, rural)

\section{Implementation of EBP}

\section{Highly influential in clinical decision making}

All oral health graduates claimed that EBP influenced what they did and how they managed to care for their patients. They could provide their patients with the evidence supporting their care, which graduates thought broadened patients' views on certain aspects of treatment. It made graduates feel powerful in "earning the trust" of patients and gave the graduates more confidence in making clinical decisions.

My clinical decisions were highly dependent on evidence when I explained properly what the treatment entailed and the research behind the proven procedure. Patients were always convinced about the clinical decision I recommended. (Participant 11, private, metro)

\section{Powerful communication tool}

The oral health graduates working in the private sector reported that current patients were looking at everything holistically. The community was inclined to want more sound evidence for any treatment planned. Evidence-based dentistry, therefore, has become a compelling communication "tool" for the graduates in providing care for their patients. Patients appeared to want the "top" science behind the proposed treatment, and they were always thankful for how the oral health graduates explained the evidence 
behind the proposed treatment. The graduates thought that patients were motivated to make some healthy changes when they were presented with convincing evidence.

A lot of young, particularly, health-conscious patients would come in with the information they found in Google and checked it out with me. (Participant 12, private, metro)

Most of the patients did their research before they came in to see me. (Participant 8 , private, metro)

When I analysed with them what was good concrete evidence and what was just hearsay, they really appreciated my knowledge to the topics. (Participant 10, private, metro)

\section{Emphasis on patient-centred care}

The oral health graduates from the private sector felt that patients trusted them more when the science behind the treatment was discussed with them and they were given treatment options according to evidence. Apart from finding the best available evidence to support treatment, oral health graduates reported that they still had to respect patients' preferences and values.

Patients always preferred the best option; that's why I based my recommendation on evidence, but sometimes cost could be an issue. (Participant 8, private, metro)

However, graduates working in the public sector reported that their patients appeared less interested in the evidence for practice but were grateful for any treatment they were provided. Such patients were apparently not critical if they were not provided with the best treatment.

Some of the patients do not show much interest in research. They did not question but agreed to whatever was provided for them. (Participant 4, public, rural)

\section{Support of EBP}

\section{Challenge by colleagues}

Graduates from the private sector perceived that dentists had mixed feelings about evidence-based practice support. Some dentists who were "up to date" with new technologies were very supportive. However, some acknowledged the emergence of new evidence but still practised what they had learned a long time ago.

Some younger clinicians in my practice would follow more on evidence. The older ones who were really set in their ways for too long, they were reluctant to accept new evidence. (Participant 1, private, metro)

The oral health graduates from the public sector reported stronger support from their managers.

There were regular team meetings to discuss current evidence and policies and procedures. In a way, this was the driving force in evidence-based practice. (Participant 3, public, rural) 


\section{Online resources are limited}

Almost all oral health graduates reported dissatisfaction with their online access to evidence from their clinical environments. Graduates from the public sector, however, were able to access the local health district intranet sites but had limited access to fulltext articles. Graduates in the private sector reported that their supervising dentists subscribed to dental journals, which gave them access to current issues; however, this did not provide any avenue to do specific searches.

We no longer had access to the University of Sydney Library for electronic resources which was kind of frustrating. (Participant 10, private, metro)

We could access the hospital medical library, but there were limited full-text articles on dentistry. (Participant 7, public, metro)

\section{Lacking evidence in some aspects}

All graduates claimed that evidence in some aspects of dentistry is limited. It was felt by graduates that a lot of research was driven by demand from of the public.

A lot of research was based on dental implants and aesthetic dentistry. There was no balance evidence in different facets of dentistry. (Participant 4, public, rural)

Some research evidence or findings were ambiguous so that it could be problematic. (Participant 11, private, metro)

\section{EBP teaching}

\section{Good critical appraisal skills}

All oral health graduates thought the EBP teaching provided in the program at the university was adequate and gave them an "edge" in being in the forefront of the oral health profession.

What we learned in our EBP lectures and tutorials was quite thorough; I was able to sift through different types of studies to determine their validity. (Participant 4, public, rural)

All the lectures and tutorials on finding the best evidence were very informative, which opened a world of knowledge to us. (Participant 2, private, metro)

Impressed by employers

The majority of the oral health graduates were very proud of how capable they were of distinguishing between "good" and "poor" evidence. Their employers were also impressed by their knowledge of how to appraising evidence.

What we learned in EBP for the Bachelor of Oral Health was of a high standard; my boss thought I was taught well in the university. I was able to explain all these terminologies in appraising research articles. (Participant 8 , private, metro) 
EBP CURRICULUM IMPACT ORAL HEALTH GRADUATES

\section{Guide for future clinical practice}

All oral health graduates indicated that they would continue integrating evidence into practice and felt this was instrumental in making clinical decisions for continuous patient care.

I would certainly continue. I would keep up to date with current evidence to make sure I am in line with standard new practice. (Participant 4, public, rural)

As people are more interested in research, I would have to keep up with all emerging evidence to guide my future clinical practice. (Participant 10, private, metro)

By comparison, the demand for sound evidence was higher in the private sector. Private patients may perceive that "pay for service" entitles them to question all treatment options. The oral health graduates working in the private sector are actively keeping up to date. Public patients might feel that their only option is to trust and be grateful for what was provided, as the demand for sound evidence was not so great. There was a system in place to keep the clinicians in the public sector up to date.

By using triangulation, the validity of conclusions is enhanced through testing the consistency of the findings from different research components conducted in series; this helped provide us with a broader understanding of our research topic (Farmer et al., 2006). Thus, the results included students' EBP experience, as measured by the questionnaire on integrating EBP in student clinical practice (G. Wong et al., 2018); qualitative evidence of students' EBP clinical decision-making process, from the focus group; and the impact of the EBP curriculum on graduates' career practice, from the individual interviews. The diversity of methods not only cross-referenced data but also provided a greater depth of analysis of different perspectives (Carter et al., 2014). Table 2 shows a summary of the main findings from the questionnaire together with the themes identified from student focus groups and graduate individual interviews. The "•" in relevant columns indicates that a theme was identified in that research component. There were consistent findings across the three components. From the initial questionnaire, all students agreed that EBP positively influenced their clinical decisions. The focus groups found learning EBP provided a "sound foundation to clinical decision-making" and helped "consolidate learning". The graduates described implementing EBP in their career practice as a "powerful communication tool" that was "highly influential in the clinical decision". From the questionnaire, most students (90\%) indicated their acceptance of integrated EBP as part of clinical practice and were confident $(80 \%)$ in EBP application. The oral health graduates echoed this finding, quoting EBP in practice as "building confidence" (Participant 5, public, rural) while also referring to it as "a guide for future practice" (Participant 10, private, metro). This integration and triangulation of findings from three different components identified areas of overlap and demonstrated further depth in the graduates' perception of the impact of an EBP curriculum on their career practices. 
Table 2

Summary of Themes Identified in the Different Components of Research

\begin{tabular}{|c|c|c|c|}
\hline & Questionnaire & Focus Group & Interview \\
\hline \multicolumn{4}{|l|}{ Questionnaire findings } \\
\hline EBP as an integral part of clinical practice & $\bullet$ & $\bullet$ & $\bullet$ \\
\hline EBP as part of clinical decision making & $\bullet$ & $\bullet$ & $\bullet$ \\
\hline Difficulty in evidence search and full-text access & $\bullet$ & $\bullet$ & $\bullet$ \\
\hline Confidence in critical appraisal & $\bullet$ & $\bullet$ & $\bullet$ \\
\hline Confidence in EBP application & $\bullet$ & $\bullet$ & $\bullet$ \\
\hline \multicolumn{4}{|l|}{ Themes: Student focus group } \\
\hline \multicolumn{4}{|l|}{ EBP learning experience } \\
\hline Disconnect between theory and practice & & $\bullet$ & \\
\hline Concept hard to grasp & & $\bullet$ & \\
\hline Inconsistency in tutor support across clinics & & $\bullet$ & \\
\hline Effective assessment process & & $\bullet$ & \\
\hline \multicolumn{4}{|l|}{ Facilitators and barriers in EBP } \\
\hline Constraint by full-text access & $\bullet$ & $\bullet$ & $\bullet$ \\
\hline Limited evidence in some aspects & $\bullet$ & $\bullet$ & $\bullet$ \\
\hline Inconsistent feedback & & $\bullet$ & \\
\hline Enhance learning via group discussion & & $\bullet$ & $\bullet$ \\
\hline \multicolumn{4}{|l|}{ EBP impact on clinical decision making } \\
\hline Sound foundation to clinical decision making & $\bullet$ & $\bullet$ & $\bullet$ \\
\hline Provide confidence and simulate wider perspective & & $\bullet$ & $\bullet$ \\
\hline Consolidate learning & & $\bullet$ & $\bullet$ \\
\hline Legal implications & & $\bullet$ & \\
\hline \multicolumn{4}{|l|}{ Themes: Graduate interviews } \\
\hline \multicolumn{4}{|l|}{ Perception of EBP } \\
\hline Build confidence & $\bullet$ & $\bullet$ & $\bullet$ \\
\hline Promote independent practice & & & $\bullet$ \\
\hline Vital for professional development & & & $\bullet$ \\
\hline \multicolumn{4}{|l|}{ Implementation of EBP } \\
\hline Highly influential in clinical decision & $\bullet$ & $\bullet$ & $\bullet$ \\
\hline Powerful communication tool & & & $\bullet$ \\
\hline Emphasis on patient-centred care & & & $\bullet$ \\
\hline \multicolumn{4}{|l|}{ Support of EBP } \\
\hline Challenge by colleagues & & & $\bullet$ \\
\hline Limited online resources & $\bullet$ & $\bullet$ & $\bullet$ \\
\hline Lacking evidence in some aspects & & $\bullet$ & $\bullet$ \\
\hline \multicolumn{4}{|l|}{ EBP teaching } \\
\hline Good critical appraisal skills & $\bullet$ & & $\bullet$ \\
\hline Impressed by employers & & & $\bullet$ \\
\hline Guide for future clinical practice & & & $\bullet$ \\
\hline
\end{tabular}




\section{Discussion}

There are numerous studies documenting EBP teaching for medical, nursing and health students. Kyriakoulis et al.'s (2016) systematic review reported that multiple interventions are better than a single intervention. An increase in the duration and frequency of the interventions may result in longer knowledge retention. Convergent results are found in our study. The multifacted approach, incorporating scenario-based learning (SBL) tutorials linked to clinical practice, integrating EBP in student clinical practice and introducing a progressive assessment strategy to improve student learning through providing formative feedback to students, has proven effective in building oral health graduates' confidence in applying EBP in their career practice.

Integrating EBP into the undergraduate oral health curriculum improved knowledge and skills in the application of EBP. However, possessing the skill conceptually does not necessarily mean it becomes intrinsically practised in the clinic. One can never assume that education means practical action (McCluskey \& Lovarini, 2005). A study by McCluskey and Lovarini (2005) investigated the EBP behaviour of a group of occupational therapists after an EBP education workshop. The changes in behaviour were small, and the authors called for education focused on helping learners to establish routines and priorities around EBP. While the knowledge and skills of EBP can be taught, a student's attitude towards EBP cannot be entirely formed by the curriculum. The routine clinical behaviour of applying EBP, which can be referred to as the "culture", has to be nurtured rather than "taught" (Dawes et al., 2005; Marinho et al., 2001). The organisational culture needs to support and sustain evidence-based practice (Austin \& Ciaassen, 2008; Dawes et al., 2005; Marinho et al., 2001).The use of evidence is ideally reflected in day-to-day professional attitude and practice as an evidence-seeking behaviour. There are multi-dimensional factors influencing health professionals to fully adopt EBP behaviour, such as motivations, capabilities, environmental context and resources (McCluskey \& Lovarini, 2005). The environmental context plays a vital role in establishing an EBP culture (Hinton et al., 2011). The oral health graduates who worked in the public sector reported stronger support from their colleagues, such as dentists and other oral health professionals in the team. However, the graduates who worked in the private sector were challenged by a culture of low evidence use, as some practitioners were not so receptive to current research. Perhaps they see the new evidence as a threat to the currency of their professional practice. Apart from integrating EBP in student clinical practice, attention should also be paid to raising the EBP self-efficacy of professionals in the field, as suggested by Spek, Wieringa-de Waard, Lucas, \& van Dijk's (2013) study. Perhaps the new oral health graduates working in the private sector can drive a culture change by encouraging the use of evidence to promote clinical effectiveness for the profession. There are institutional systems in place to make sure that the clinicians in the public sector are up to date, such as regular journal club meetings and performance reviews. Graduates working in the public sector may, thus, have more opportunity to adopt and sustain the use of EBP in the profession. All health professionals are obliged to provide evidence-based patient-centred care. Therefore, it is necessary to ensure that dentistry and oral health remains a scientifically-driven health 
profession. Since 2013, the dental accreditation standards in the United States and Canada have called for dental schools to integrate EBP into curricula. The Australian Dental Council (2016) has identified EBP as one of the competency standards required for oral health professionals since early 2016. Many oral health graduates volunteered for the research interviews in this study, demonstrating their enthusiasm for EBP. However, their motivation can be dampened by constraints in the environmental context and by lack of resources. This finding is consistent with a study by Kishore et al. (2014), which reported that contrary to the situation in medicine, relatively few randomised controlled trials existed in dentistry to compare the outcome measures of clinical interventions.

Access to research evidence is a significant challenge. Library access in clinical settings may also engage clinicians in applying research findings to clinical practice (Kulier, Gee, \& Khan, 2008). In our study, graduates in the private sector reported that they struggled to get digital access to search engines, let alone full-text journal articles. This finding is consistent with the barriers faced by nurses in the United States (BekeHarrigan, Hess, \& Weinland, 2008; Fink, Thompson, \& Bonnes, 2005; Melnyk et al., 2004; Ratner, 2006) and speech and language therapists in Ireland and other countries (O'Connor \& Pettigrew, 2009; Ratner, 2006). Though PubMed provides free access, as a government-sponsored system that searches the MEDLINE database, it has limited free access to full-text articles (S. S. Wong, Wilczynski, \& Haynes, 2006). It is not a system that efficiently deals with accurate searching, so it can return articles of limited relevance. Purchase of full-text articles online is an option, but graduates described the cost as an issue, which is consistent with Pravikoff, Tanner, \& Pierce's (2005) study. It was pleasing to hear that graduates reported that some dental practitioners supported $\mathrm{EBP}$ in the private sector by subscribing to dental journals, which is conducive to the EBP culture of continuous professional development. This practice, however, did not solve the problem of searching for evidence required to answer their specific clinical questions. Graduates from the public sector have better EBP support at the organisation level. Oral health practitioners are provided access to the Cochrane Library via the Clinical Information Access Portal (CIAP). With this organisation support to promote EBP, patient-centred care can be optimised (Tagney \& Haines, 2009).

In order to endorse EBP in the clinical environment, providing the ability to access robust evidence is essential. The types of resources available certainly impact the clinical decision-making process. The University of Sydney oral health graduates reported that evidence resources were driven by demand by the public, such as aesthetic dentistry and dental implants, and so evidence in some other aspects of dentistry was limited. Some research findings were ambiguous, and the lack of action points was problematic in EBP application. This is consistent with Kishore et al.'s (2014) recommendation that evidence needed to have a broad range of outcomes that are considered important by patients.

EBP offers a framework for problem solving and clinical decision making for oral health professionals, however there are still challenges to the adoption of EBP and sustained evidence-seeking behaviour in the oral health profession. Based on the findings from 
this current research, digital access to high-quality evidence journals for oral health graduates would be a sound investment towards building an EBP culture.

\section{Conclusion}

In this study, most oral health graduates from the the University of Sydney perceived a good standard of EBP teaching was a vital part of professional clinical practice. Graduates felt confident and empowered by the process of making clinical decisions based on sound evidence. However, online resources for literature search and evidence in some aspects of dentistry could be limited. Increased access to full-text evidence would, undoubtedly, overcome significant barriers to implementing EBP. The EBP curriculum in the Bachelor of Oral Health program made a positive impact on oral health graduates' career practice and supported the continual integration of EBP in the curriculum. This study reveals a successful implementation of an EBP program in clinical practice that may be applicable to other healthcare educational institutions and provides evidence for supporting translational science by bridging the gap between research and clinical practice.

\section{Further research and implications}

This research explored oral health students' perceptions of their EBP learning experience and the initial impact of EBP on clinical decision making 12 months after graduation. Future research should investigate if the graduates continue using scientific evidence for clinical practice and how their perceptions change over time. A larger scale study, possibly at the national level, could give an account of the methodology used for teaching EBP in other existing undergraduate oral health curricula in other universities and its impact on the profession. Additionally, a cross-comparison study investigating the difference in EBP application to practice between oral health and other allied health professionals would be warranted.

Strategies are needed to escalate changes at an organisational level to enhance oral healthcare outcomes by testing implementation interventions collectively. Medicine and nursing are at the forefront of translational science and EBP in the United States; perhaps dentistry and oral health can take a similar approach in implementing and sustaining EBP on a bigger scale in organisations (Titler, 2014). To inform oral health practitioners about clinical outcomes, other stakeholders, and experts in bioinformatics, should be involved in tracking EBP processes by using electronic health records and collecting outcome data in clinical settings. At the same time, these clinical data could be used to test research hypotheses to provide feedback to researchers. Consequently, the gap between research and practice will be bridged and patient outcomes from patient-oriented level to population-based level will be positively improved.

\section{Acknowledgements}

The authors would like to thank the University of Sydney students and graduates who participated in this study. 


\section{Funding and conflict of interest statement}

No potential conflict of interest was reported by the authors.

\section{References}

Austin, M. J., \& Claassen, J. (2008). Impact of organizational change on organizational culture: Implications for introducing evidence-based practice. Journal of Evidence-Based Social Work, 5(1-2), 321-359. doi:10.1300/ J394v05n01_12

Australian Dental Council (ADC). (2016). Professional competencies of the newly qualitifed dental hygienist, dental therapist and oral health therapist. Retrieved from https://www.adc.org.au/sites/default/files/Media_Libraries/PDF/Accreditation/ Professional\%20Competencies\%20of\%20the\%20Newly\%20Qualified $\% 20$ Dental\%20DH\%20DT\%20OHT_rebrand\%20Final.pdf

Azarpazhooh, A., Mayhall, J. T., \& Leake, J. L. (2008). Introducing dental students to evidence-based decisions in dental care. Journal of Dental Education, 72(1), 87-109.

Beke-Harrigan, H., Hess, R., \& Weinland, J. A. (2008). A survey of registered nurses' readiness for evidence-based practice: A multidisciplinary project. Journal of Hospital Librarianship, 8(4), 440-448. doi:10.1080/15323260802382802

Carter, N., Bryant-Lukosius, D., DiCenso, A., Blythe, J., \& Neville, A. J. (2014). The use of triangulation in qualitative research. Oncology Nursing Forum, 41(5), 545-547. doi:10.1188/14.ONF.545-547

Ciarocco, N. J., Lewandowski, G. W., \& Van Volkom, M. (2013). The impact of a multifaceted approach to teaching research methods on students' attitudes. Teaching of Psychology, 40(1), 20-25. doi:10.1177/0098628312465859

Creswell, J. W. (2013). Research design: Qualitative, quantitative, and mixed methods approaches. Los Angeles, CA: Sage.

Dawes, M., Summerskill, W., Glasziou, P., Cartabellotta, A., Martin, J., Hopayian, K., . . Osborne, J. (2005). Sicily statement on evidence-based practice. BMC Medical Education, 5(1), 1.

Duffy, M. E. (1987). Methodological triangulation: A vehicle for merging quantitative and qualitative research methods. Image: The Journal of Nursing Scholarship, 19(3), 130-133.

Farmer, T., Robinson, K., Elliott, S. J., \& Eyles, J. (2006). Developing and implementing a triangulation protocol for qualitative health research. Qualitative Health Research, 16(3), 377-394.

Fink, R., Thompson, C. J., \& Bonnes, D. (2005). Overcoming barriers and promoting the use of research in practice. JONA: The Journal of Nursing Administration, 35(3), 121-129. 
Foss, C., \& Ellefsen, B. (2002). The value of combining qualitative and quantitative approaches in nursing research by means of method triangulation. Journal of Advanced Nursing, 40(2), 242-248.

Gerzina, T. M., McLean, T., \& Fairley, J. (2005). Dental clinical teaching: Perceptions of students and teachers. Journal of Dental Education, 69(12), 1377-1384.

Glasgow, R. E., \& Emmons, K. M. (2007). How can we increase translation of research into practice? Types of evidence needed. Annual Review of Public Health, 28(1), 413-433. doi:10.1146/annurev.publhealth.28.021406.144145

Gonzales, R., Handley, M. A., Ackerman, S., \& O'Sullivan, P. S. (2012). A framework for training health professionals in implementation and dissemination science. Academic Medicine, 87(3), 271-278. doi:10.1097/ACM.0b013e3182449d33

Gopalakrishnan, S., \& Ganeshkumar, P. (2013). Systematic reviews and metaanalysis: Understanding the best evidence in primary healthcare. Journal of Family Medicine and Primary Care, 2(1), 9-14. doi:10.4103/2249-4863.109934

Greiner, A. C., \& Knebel, E. (Eds.). (2003). Health Professions Education: A Bridge to Quality. Washington, DC: National Academies Press.

Grol, R. (2001). Successes and failures in the implementation of evidence-based guidelines for clinical practice. Medical Care, 39(8, Suppl. 2), 46-54.

Hinton, R. J., Dechow, P. C., Abdellatif, H., Jones, D. L., McCann, A. L., Schneiderman, E. D., \& D’Souza, R. (2011). Creating an evidence-based dentistry culture at Baylor College of Dentistry: The winds of change. Journal of Dental Education, 75(3), 279-290.

Kersten, H. W., Vervoorn, J. M., Zijlstra, A. E., Blok, B. S., \& van Eijden, T. M. (2007). Development and implementation of new educational concepts in a dental curriculum. European Journal of Dental Education, 11(1), 2-9.

Kishore, M., Panat, S. R., Aggarwal, A., Agarwal, N., Upadhyay, N., \& Alok, A. (2014). Evidence based dental care: Integrating clinical expertise with systematic research. JCDR: Journal of Clinical and Diagnostic Research, 8(2), 259-262. doi: $10.7860 / J C D R / 2014 / 6595.4076$

Kulier, R., Gee, H., \& Khan, K. S. (2008). Five steps from evidence to effect: Exercising clinical freedom to implement research findings. BJOG, 115(10), 1197-1202. doi:10.1111/j.1471-0528.2008.01821.x

Kyriakoulis, K., Patelarou, A., Laliotis, A., Wan, A. C., Matalliotakis, M., Tsiou, C., \& Patelarou, E. (2016). Educational strategies for teaching evidence-based practice to undergraduate health students: Systematic review. Journal of Educational Evaluation for Health Professions, 13, 34. doi:10.3352/jeehp.2016.13.34

Legare, F., Ratte, S., Gravel, K., \& Graham, I. D. (2008). Barriers and facilitators to implementing shared decision-making in clinical practice: Update of a systematic review of health professionals' perceptions. Patient Education \& Counseling, 73(3), $526-535$. 
Long, K. A. (2003). The Institute of Medicine report: Health professions education. A bridge to quality. Policy, Politics, \& Nursing Practice, 4(4), 259-262. doi:10.1177/1527154403258304

Marinho, V. C., Richards, D., \& Niederman, R. (2001). Variation, certainty, evidence, and change in dental education: Employing evidence-based dentistry in dental education. Journal of Dental Education, 65(5), 449-455.

McCluskey, A., \& Lovarini, M. (2005). Providing education on evidence-based practice improved knowledge but did not change behaviour: A before and after study. BMC Medical Education, 5(1), 1.

Melnyk, B. M., Fineout-Overholt, E., Fischbeck Feinstein, N., Li, H., Small, L., Wilcox, L., \& Kraus, R. (2004). Nurses' perceived knowledge, beliefs, skills, and needs regarding evidence-based practice: Implications for accelerating the paradigm shift. Worldviews on Evidence-Based Nursing, 1(3), 185-193. doi:10.1111/j.1524-475X.2004.04024.x

Miles, A., Loughlin, M., \& Polychronis, A. (2007). Medicine and evidence: Knowledge and action in clinical practice. Journal of Evaluation in Clinical Practice, 13(4), 481-503. doi:10.1111/j.1365-2753.2007.00923.x

O'Connor, S., \& Pettigrew, C. M. (2009). The barriers perceived to prevent the successful implementation of evidence-based practice by speech and language therapists. International Journal of Language \& Communication Disorders, 44(6), $1018-1035$.

Palinkas, L. A., Horwitz, S. M., Green, C. A., Wisdom, J. P., Duan, N., \& Hoagwood, K. (2015). Purposeful sampling for qualitative data collection and analysis in mixed method implementation research. Administration and Policy in Mental Health, 42(5), 533-544. doi:10.1007/s10488-013-0528-y

Pravikoff, D. S., Tanner, A. B., \& Pierce, S. T. (2005). Readiness of U.S. nurses for evidence-based practice. The American Journal of Nursing, 105(9), 40-51.

Ratner, N. B. (2006). Evidence-based practice: An examination of its ramifications for the practice of speech-language pathology. Language, Speech, and Hearing Services in Schools, 37(4), 257-267. doi:10.1044/0161-1461(2006/029)

Sackett, D. L., Rosenberg, W. M., Gray, J. A., Haynes, R. B., \& Richardson, W. S. (1996). Evidence based medicine: What it is and what it isn't. BMJ, 312(7023), $71-72$.

Saint, D. A., Horton, D., Yool, A., \& Elliott, A. (2015). A progressive assessment strategy improves student learning and perceived course quality in undergraduate physiology. Advances in Physiology Education, 39(3), 218-222.

Sargeant, J. (2012). Qualitative research part II: Participants, analysis, and quality assurance. Journal of Graduate Medical Education, 4(1), 1-3. doi:10.4300/ JGME-D-11-00307.1

Satterfield, J. M., Spring, B., Brownson, R. C., Mullen, E. J., Newhouse, R. P., Walker, B. B., \& Whitlock, E. P. (2009). Toward a transdisciplinary model of evidence-based practice. Milbank Quarterly, 87(2), 368-390. 
Schardt, C. (2011). Health information literacy meets evidence-based practice. Journal of the Medical Library Association, 99(1), 1-2.

Seçkin, G., Yeatts, D., Hughes, S., Hudson, C., \& Bell, V. (2016). Being an informed consumer of health information and assessment of electronic health literacy in a national sample of internet users: Validity and reliability of the e-HLS instrument. Journal of Medical Internet Research, 18(7), e161-e161. doi:10.2196/jmir.5496

Smith, D. W. (2018). Phenomenology. In E. N. Zalta (Ed.), The Stanford Encyclopedia of Philosophy (Summer 2018 ed.). Retrieved from https://plato.stanford.edu/ archives/sum2018/entries/phenomenology/

Spek, B., Wieringa-de Waard, M., Lucas, C., \& van Dijk, N. (2013). Teaching evidence-based practice (EBP) to speech-language therapy students: Are students competent and confident EBP users? International Journal of Language \& Communication Disorders, 48(4), 444-452.

Starks, H., \& Trinidad, S. B. (2007). Choose your method: A comparison of phenomenology, discourse analysis, and grounded theory. Qualitative Health Research, 17(10), 1372-1380.

Tagney, J., \& Haines, C. (2009). Using evidence-based practice to address gaps in nursing knowledge. British Journal of Nursing, 18(8), 484-489.

Teddlie, C., \& Tashakkori, A. (2011). Mixed methods research: Contemporary issues in an emerging field. In N. K. Denzin \& Y. S. Norman (Eds.), The Sage handbook of qualitative resrearch (4th ed.) (pp. 285-300). Thousand Oaks, CA: Sage.

Teich, S. T., Demko, C. A., \& Lang, L. A. (2013). Evidence-based dentistry and clinical implementation by third-year dental students. Journal of Dental Education, 77(10), 1286-1299.

Titler, M. G. (2014). Overview of evidence-based practice and translation science. Nursing Clinics of North America, 49(3), 269-274.

Vranceanu, A.-M., Cooper, C., \& Ring, D. (2009). Integrating patient values into evidence-based practice: Effective communication for shared decision-making. Hand Clinics, 25(1), 83-96. doi:https://doi.org/10.1016/j.hcl.2008.09.003

Werb, S. B., \& Matear, D. W. (2004). Implementing evidence-based practice in undergraduate teaching clinics: A systematic review and recommendations. Journal of Dental Education, 68(9), 995-1003.

Wong, G., Monatzerolghaem, M., \& Gerzina, T. (2018). Integrating evidencebased practice into oral health clinical practice: Students' perspectives. Journal of Evidence-Based Dental Practice, 19(1), 79-85. doi:10.1016/j.jebdp.2018.11.002

Wong, S. S., Wilczynski, N. L., \& Haynes, R. B. (2006). Comparison of topperforming search strategies for detecting clinically sound treatment studies and systematic reviews in MEDLINE and EMBASE*. Journal of the Medical Library Association, 94(4), 451.

Young, T., Rohwer, A., Volmink, J., \& Clarke, M. (2014). What are the effects of teaching evidence-based health care (EBHC)? Overview of systematic reviews. PloS one, $9(1)$. 